\title{
WiMAX network performance monitoring \& optimization
}

\author{
Zhang, Qi; Dam, H
}

Published in:

IEEE Network Operations and Management Symposium, 2008. NOMS 2008.

Link to article, DOI:

10.1109/NOMS.2008.4575179

Publication date:

2008

Document Version

Publisher's PDF, also known as Version of record

Link back to DTU Orbit

Citation (APA):

Zhang, Q., \& Dam, H. (2008). WiMAX network performance monitoring \& optimization. In IEEE Network Operations and Management Symposium, 2008. NOMS 2008. IEEE.

https://doi.org/10.1109/NOMS.2008.4575179

\section{General rights}

Copyright and moral rights for the publications made accessible in the public portal are retained by the authors and/or other copyright owners and it is a condition of accessing publications that users recognise and abide by the legal requirements associated with these rights.

- Users may download and print one copy of any publication from the public portal for the purpose of private study or research.

- You may not further distribute the material or use it for any profit-making activity or commercial gain

- You may freely distribute the URL identifying the publication in the public portal

If you believe that this document breaches copyright please contact us providing details, and we will remove access to the work immediately and investigate your claim. 


\title{
WiMAX Network Performance Monitoring \& Optimization
}

\author{
Qi Zhang \\ qz@com.dtu.dk \\ Communication, Optics \& Materials department \\ Technical University of Denmark \\ Henrik Dam \\ hd@dansketelecom.com \\ DanskeTelecom
}

Danske Telecom COM・DTU

\begin{abstract}
In this paper we present our WiMAX (Worldwide Interoperability for Microwave Access) network performance monitoring and optimization solution. As a new and small WiMAX network operator, there are many demanding issues that we have to deal with, such as limited available frequency resource, tight frequency reuse, capacity planning, proper network dimensioning, multi-class data services and so on. Furthermore, as a small operator we also want to reduce the demand for sophisticated technicians and man labour hours. To meet these critical demands, we design a generic integrated network performance monitoring and optimization solution which includes traffic monitor and analyzer, dynamic quality of service control tool, radio signal quality monitor, CPE mobility tracking, interference monitor \& analyzer and optimization tool, report generator and alarm management tool. We develop and implement this integrated network performance monitoring and optimization system in our WiMAX networks. This integrated monitoring and optimization system has such good flexibility and scalability that individual function component can be used by other operators with special needs and more advanced function components can be developed in the future. The usage of system has proven that it can reduce both CAPEX (capital expense) and OPEX (operational expense), meanwhile it can improve end-to-end quality of service.
\end{abstract}




\section{Outline}

\section{Background Introduction}

Motivation

\section{WiMAX network performance monitoring \& optimization} system

$>$ Solution overview

Function components introduction

- Traffic monitor and analyzer

- Dynamic Quality of Service Control

- Radio signal quality monitor \& CPE mobility tracking

- Interference monitor \& radio, frequency planning optimization

- Report Generation \& Alarm management

\section{Benefits}

This paper is organized as following. It is mainly composed of four parts. First of all, we give a short introduction of the challenges and demands faced by the new and small wireless broadband network operator. We explain the motivation of designing and implementing an integrated WiMAX network performance monitoring and optimization system in the second part. The detailed function components in the monitoring and optimization system are presented individually in third part. The main function components consist of traffic monitor, dynamic quality of service control tool, radio signal quality monitor \& CEP mobility monitor, interference monitor and radio, frequency planning optimization, report generator and alarm management tool. These function components are integrated and interact, for example, the outputs of one function component can be the inputs of another component. Finally we will conclude the paper by giving the benefits of the network performance management system. 


\section{Background Introduction}

Differences between wireless voice based networks and wireless broadband networks:

Asymmetrical bandwidth and traffic flows

Broadband packet switched, i.e. bursty traffic, statistical properties other than Erlang

Multi-type customers : Different SLAs

Multi-Class data services: Satisfactory service level depends on the service, requiring resource allocation and QoS control on different applications

- Demanding issues in a Wireless Broadband Network, seen from the operator's perspective

$>$ Tight Frequency Reuse

$>$ Optimize Spectral Efficiency

$>\mathrm{CPE}$ Characteristics and Users Behavior (usage, modulation, mobility)

$>$ End to end quality

$>$ Network dimensioning

- Calculation and control overbooking factor and blocking probability for multi-class traffic

WiMAX is a new last mile wireless broadband access network as an alternative to wired broadband such as cable model and DSL. At the same time WiMAX is becoming a good competitor of $3 \mathrm{G}$ networks. It is a very promising broadband wireless network technology. On the other hand, WiMAX operators are facing a big challenge in WiMAX network planning and operation. The reasons lie in that WiMAX should be able to provide multi-class data services and voice service with different QoS (Quality of Service) requirements. The diversity of services and customer behavior has significant impact on the traffic pattern. Therefore, the capacity demand estimation can not be performed by traditional voice based wireless network. Furthermore, due to mobility function in WiMAX, customers are able to access network at any place within our network coverage. Different locations of a customer often result in variations of modulation performance, which eventually results in the variation of the whole network's capacity performance. Hence, the capacity planning is becoming thorny.

The customer population density variation also brings challenges for radio planning. The size of the cell varies according to the population density: It is limited by the capacity demand in high population density area and it depends on coverage limitation in low population density area. Thus it brings about challenges in frequency planning because of cell size variation and the limited available frequency.

As a network operator we provide a variety of service products with different SLAs (Service Level Agreement) to meet the different customers' need, which makes it necessary for us to provide an efficient and dynamic end-to-end quality control scheme in the network. 


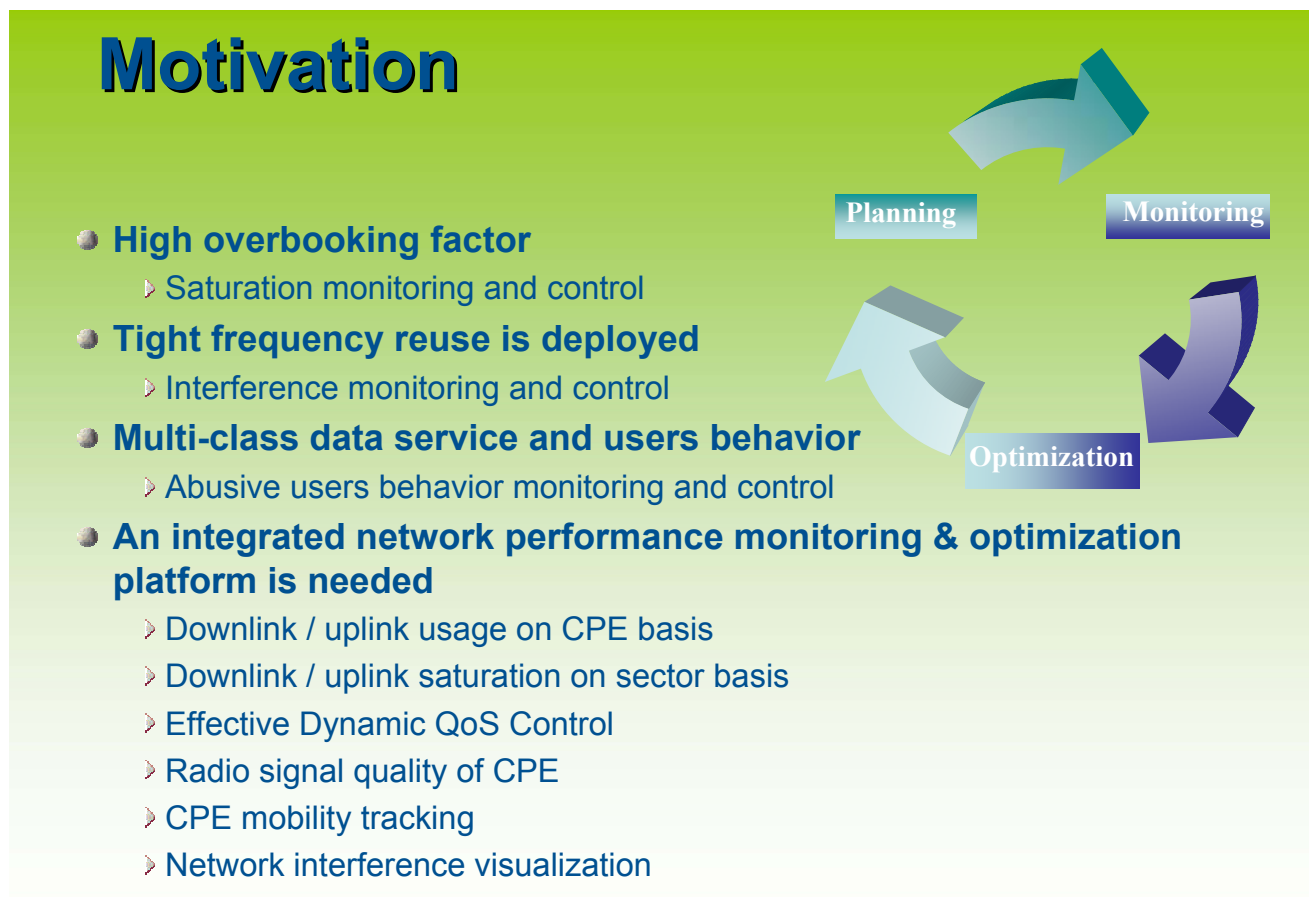

IEEE//IFIP NOMS 2008 Danske Telecomे COM・DTU

Due to the limited funds for the roll-out network, new wireless broadband network operators want to keep a high overbooking factor to reduce the equipment cost, i.e., CAPEX. Meanwhile we have to ensure good objective quality of service and customer subjective online experience based on their SLAs. Both over network provisioning and under network provisioning are costly. According to our network operation experience and experiments we find that saturation of the cell is the main cause of customers' bad online experience. Therefore it is necessary to monitor the traffic usage of the network and react to prevent network from getting into saturation.

Based on traffic usage analysis, we find the saturation in a sector is often caused by a few abusive customers. How to deal with these heavy users with minimum impact on customer contract? How to react to the network problem at a right time? To fulfil all these requirements, effective dynamic QoS control model is brought into the system.

Due to the limited frequency available for WiMAX network operator, precise network planning and tight frequency reuse is deployed for the roll-out network. For example we generally use 1:4 frequency reuse factor but in some spots 1:1 frequency reuse pattern is also used. To ensure no user suffers service degradation due to interference, it is very necessary to monitor the interference performance and to suppress interference. Inference monitoring results is a good input for optimization of radio and frequency planning.

We use planning, monitoring and optimization design cycle to implement the integrated WiMAX network management system. 


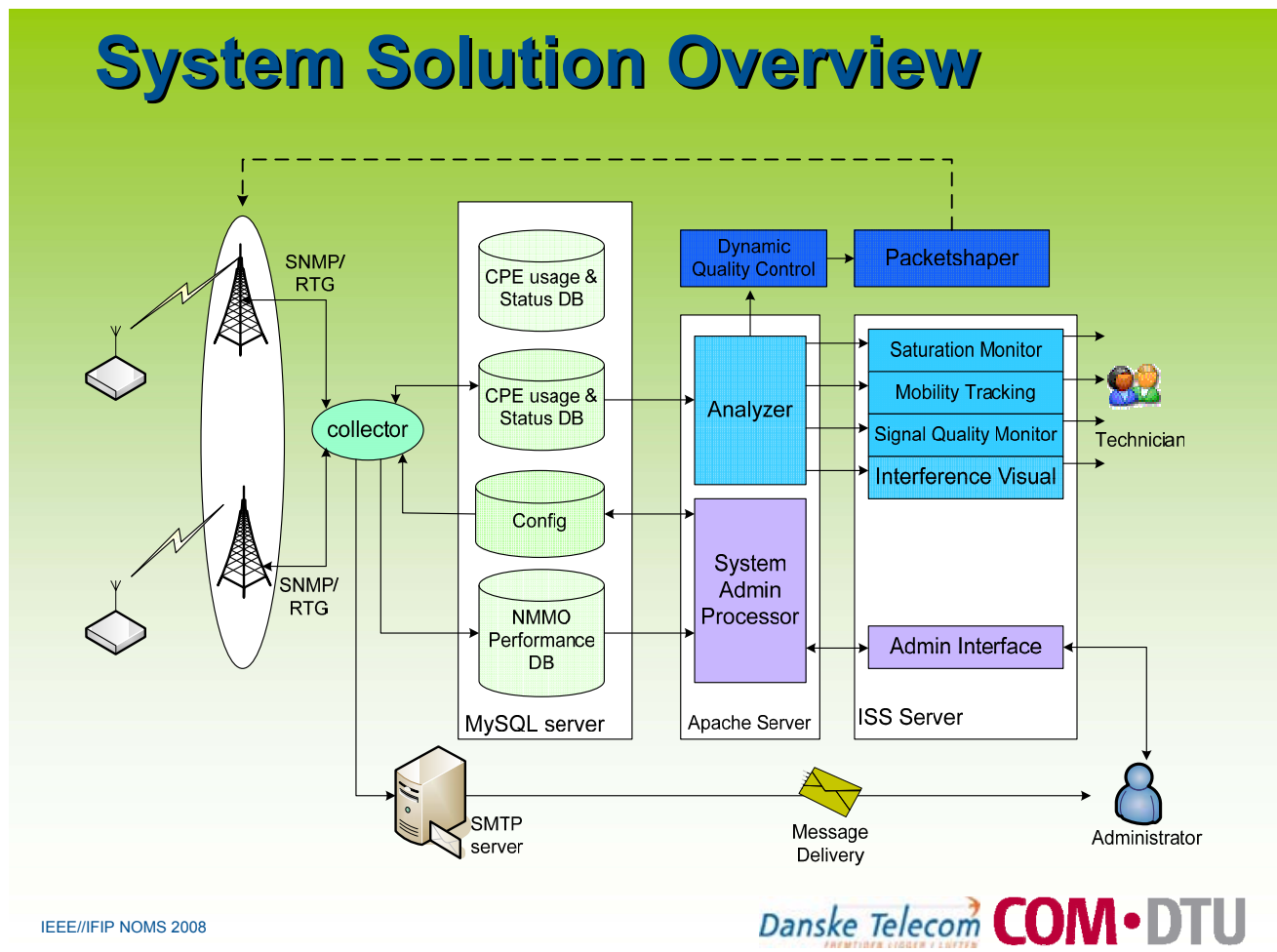

WiMAX network monitoring and optimization system can be divided into six main function blocks:

- Collector: There are two approaches to collect the network performance statistics. One is implemented by RTG (Real Traffic Grabber) or similar SNMP (Simple Network Management Protocol) based collection routine. The other is implemented by Telnet.The collection period varies depending on the different performance statistics characteristics.

- Storage: The collected data are all stored with a time stamp in MySQL database. In the storing data procedure, there is need to perform a sort of pre-processing the raw data from the SNMP agents located in the Base Stations. It is important to provide generic statistics format for different statistics analyzers. The storage block also consists of configuration database and a database containing the performance of the WiMAX network monitoring and optimization system.

- Processing: It is a procedure to analyze the collected data in the database. All the processing functions are developed by Java program.

- Visualization: It is user interface for our customer support technicians. The visualization should be designed to be able to give a clean and clearly picture of the network performance or a straightforward network problem indication.

- Control and optimization: It is the feedback action or control policy executed by control function components, when the network has problems, for instance, severe saturation.

- Administration: The administrator is capable to configure the system parameters, for example, different data collection period, saturation threshold, the control policy and so on.

In the following we will present the function components in our WiMAX network performance monitoring and optimization system. 


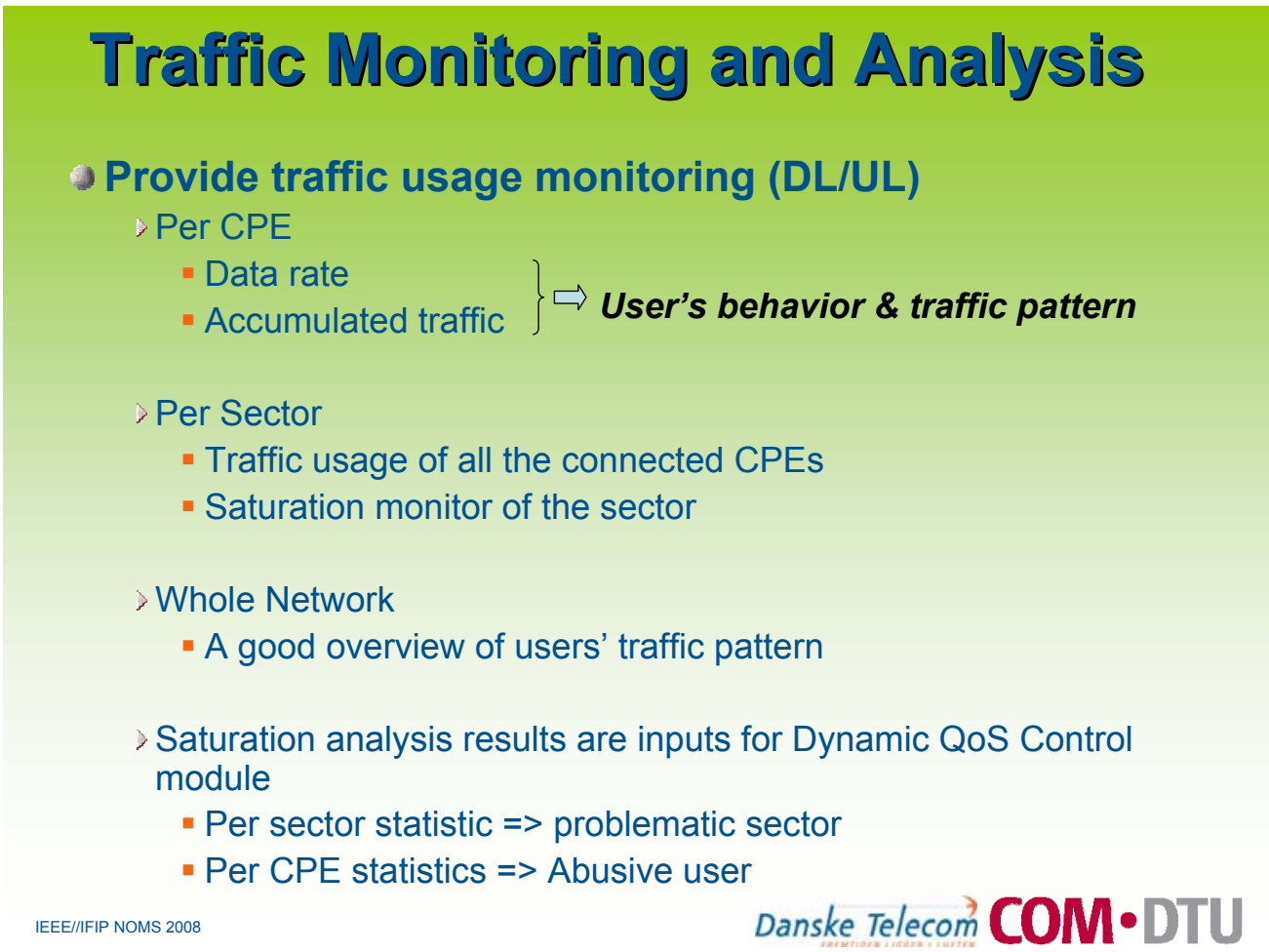

To run a fast growing roll-out network, it is of great importance to estimate precisely the capacity demand. Traffic monitoring and analysis toolbox monitors traffic usage both on downlink and uplink, which is designed to meet the need. Traffic monitoring and analysis tool differentiates on three levels, on CPE basis, sector basis or whole network basis. The monitoring of per CPE traffic usage records the data rate of CPE and the accumulated traffic. The statistical characteristics of the data rate and accumulate traffic can be used for analyzing users' behaviour and traffic pattern. The traffic usage monitoring results of each sector is used to analyze sector saturation status. It can show the time that the sector is saturated presented in CDF (Cumulative Distribution Function) graph. The saturation threshold is configured and adjusted by system administrator.

Based on our experience and experiment, we find that many users' bad online experience is often resulted from saturation in the network which is caused just by few abusive users. To solve this crucial issue, we design Dynamic QoS Control (toolbox) which will be addressed in the following slides. So the traffic usage monitoring and analysis results are good input for dynamic QoS control toolbox. The sector level saturation analysis results are used to find the problematic sector, and the CPE level traffic usage analysis results are used to find the abusive users. The severe saturation status can trigger DQC automatically to execute dynamic quality control strategy on the abusive users. 


\section{Dynamic QoS Control}

\section{Problem attacked by Dynamic QoS Control module}

$>$ Fraction of customers go against statistics, e.g.

- Use peer to peer software

- Stream music/ video...

$>$ Saturation on base station

- Deteriorate experience of the customers in the BS

- Decrease the overbooking factor

\section{- Conventional solution is inefficient}

$>$ Inspecting and classifying customers manually

Slow and error prone process requiring many man hours

$>$ Big delay of solving problems

$>$ Abusers are easily forgotten

Classification far from fair

$>$ Impossible to keep up the network growing speed.

Dynamic QoS Control toolbox aims to attack the problem caused by few users who use a lot of heavy load applications. For example, peer to peer application results in high upload traffic which does not follow normal network traffic. The network is designed by supposing that the network has asymmetrical traffic (more download traffic than uplink). When many aggressive peer to peer applications are used in the network, the network will easily come into uplink saturation. Uplink saturation will not only result in user upload speed but also in turn result in increasing downlink response time and limiting the download speed and utilization.

The saturation in the base station deteriorates users' experience in the base station. If we want to guarantee QoS of the users and at the same time we cannot do any control reaction, only small overbooking factor can be used in the network.

Conventionally technicians tried to solve the problem by inspecting and classifying users manually. It not only costs a lot of man hours but also the whole process is very slow and not accurate. Such process results in big delay of solving such problem due to network saturation. Because of delay, the abusers are easily forgotten. Moreover, there is not reasonable rule to classify abusers from normal users, so the classification is lack of fairness. In belief, the conventional solution is not scalable and it is impossible to keep up with the network growing speed. Therefore, a more efficient traffic control toolbox is in need. Thus dynamic QoS control toolbox comes into the picture.

The goal of the dynamic QoS control (DQC) toolbox is to improve customer online experience, furthermore to keep the high overbooking factor in the network. To achieve the goal, DQC should be able to isolate heavy users but allow users to have occasional heavy use. The DQC should not impact customer contract terms, in other words, 


\section{Dynamic QoS Control (cont)}

- Goal of DQC

$>$ Improve customer online experience

$>$ Increase network-wide overbooking factor

\section{Design requirements of DQC}

$>$ Isolate heavy users, allowing occasional heavy use

$>$ Impact individual customer contract terms minimally

$>$ Minimize delay between network problems and DQC responses

\section{- DQC solution}

$>$ Detect problematic sectors (BSs)

$>$ Find the abusive users in the problematic sectors

$>$ Individual customer policies applied to the abusive users

DQC strategy should take account of individual customer contact. The delay between network problem and DQC responses should be minimized, on the other hand, DQC should not be overreact. Therefore, DQC should be able to judge intelligently whether the network has problem and when DQC can react.

The solution of DQC is as following. First of all, it needs to detect problematic sectors, which is achieved by using sector saturation analysis results from traffic usage monitoring toolbox. The sector saturation CDF (cumulated distribution function) graph describes how many percentage of a given time duration, a sector is saturated over a saturation threshold. For instance, when a sector has been experiencing saturation more than $90 \%$ of the given time duration, it is marked as problematic sector. Then an upcoming question is how long the CDF time duration should be. It can not be too long or too short, because long time duration results in potential DQC reaction delay to the network problem and slow statistics feedback from the network, on the other hand, short time duration will cause DQC to overreact. Therefore, the solution is based on both long-term and short-term CDF analysis results to detect a problematic sector.

In a problematic sector, usually the users are regarded as abusive users if their accumulated traffic load has exceeded their contract and they still have high traffic usage demand.

After sorting out the abusers from the problematic sector, individual customer policies are applied to the customers. According to customer SLA, a set of increasingly hard shaping rules is designed. The shaping rules are applied on the abusers individually by an iterative approach. The basic idea is that any abuser is shaped based on the user's current maximal data rate. If saturation has not been solved after one shaping cycle, DQC will choose to use the 


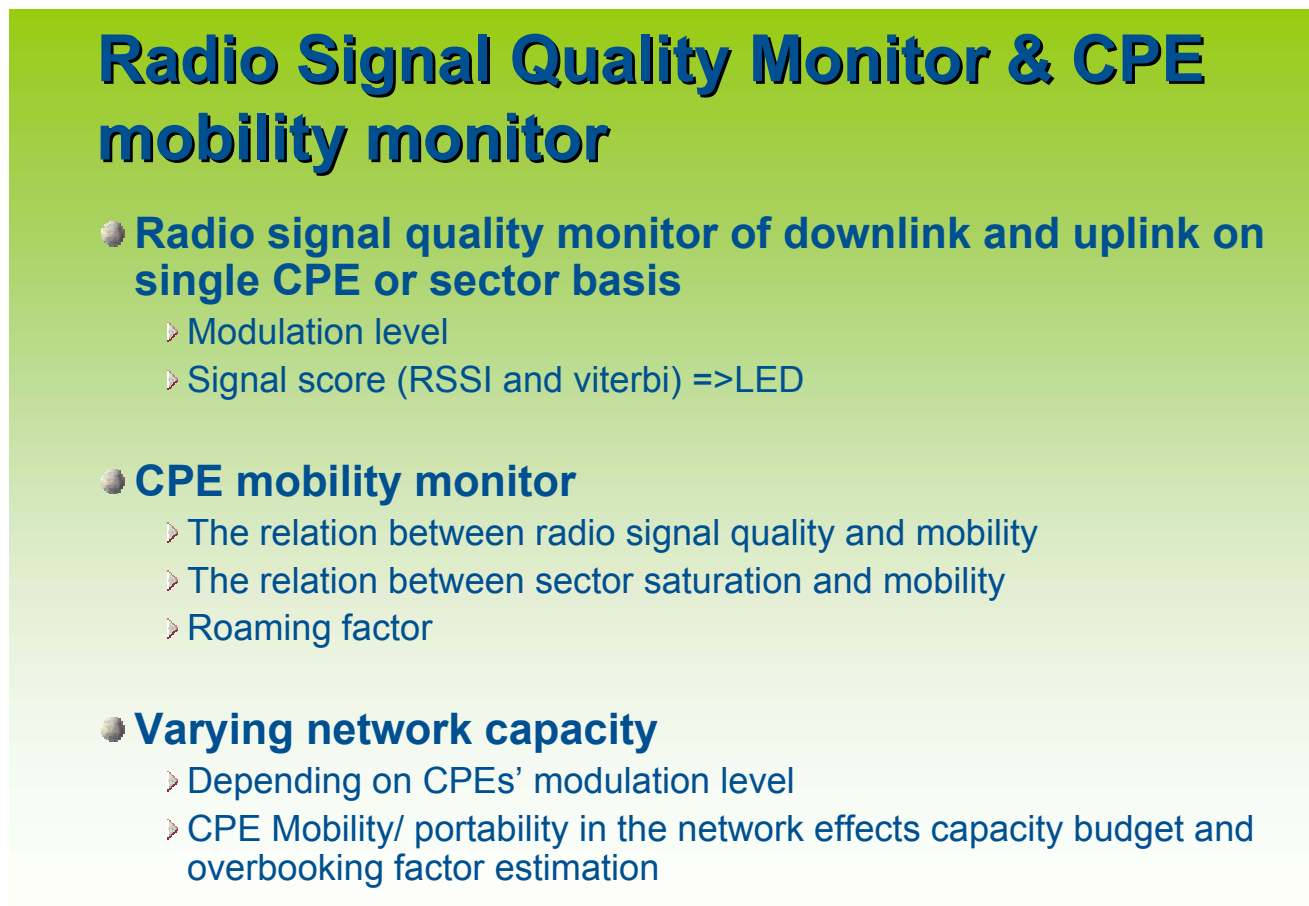

IEEE//IFIP NOMS 2008 Danske Telecomे COM・DTU

higher level shaping rules until there is no saturation.

Besides saturation resulting in user bad online experience, poor radio signal quality is another reason. So it is necessary to monitor all CPEs radio signal quality on both downlink and uplink link. It gives a very straight-forward picture of what about the modulation level and signal score (calculated by RSSI and viterbi code) of each CPE and the average value in each sector.

CPE mobility tracking is also an interesting function. The roaming of CPE has three main possibilities: 1. Due to the portable capability of the WiMAX CPE, it can be moved by user physically at large distance for instance from one city to another; 2. Due to small distance movement of CPE resulting in radio signal degration, for instance from one room to another, the $\mathrm{CPE}$ can register to another neighbouring sector; 3 . When the home sector is in saturation and the CPE starts initial ranging, the home sector BS will recommend the CPE to roam to another BS. A roaming factor is defined as the sum of CPEs in all base station divided by the sum of CPEs in the whole network.

$$
R F=\frac{\sum_{i=1}^{N} \sum_{B S=i} \text { UniqueCPEs }}{\sum_{N E T} \text { UniqueCPEs }}
$$

The roaming factor can be used to estimate the amount of mobility / portability in the network, furthermore in the business analysis it is used for estimating the revenue contribution from the individual base station.

Additionally, the WiMAX network capacity is a kind of varying capacity which depends on the modulation and coding scheme used by the CPEs. So the real-time CPE radio signal quality results can be used to calculate the real-time network capacity. The more accurate network capacity planning can obtain by taking account of the 


\section{Interference Monitor, Analyzer and Network Optimization}

\section{Interference collector}

Collect interference data from base station

Accumulate network interfered symbol amount

Differentiate Interference symbols by received power of the symbols

co-channel interference monitor and analyzer

Prioritize the sectors according to the severity

$>$ Get an overview over where the interfering CPEs are connected.

- Visualization for the interfered sector

$>$ Interference map with Interference source status

- Optimization

$>$ Based on the amount of symbols, signal strength and location of the individual CPEs, to choose the best optimization method, i.e. tilt antennas, optimize frequencies etc...

impact of CPE's mobility / portability.

Poor radio connection often results in high data error rate, frequent retransmission, bad throughput performance and user unpleasant online experience. The reason of poor radio signal quality lies in that radio signal suffers severe path loss due to long distance between BS and CPE or bad location of CPE, or there is strong interference. There are uplink and downlink interference. For the first phase, the uplink interference monitor and analyzer has been implemented, which is an effective tool for technicians to do troubleshooting of bad radio connection. The downlink interference monitor should be at each $\mathrm{CPE}$, which is under investigation.

The interference collector is implemented based on the idea that the base stations use idle time to listen to co-channel CPEs that are connected in the other base stations in the network. It can record all the number of interference symbols with different signal strength. The interference collector collets these interference data once a day and store them in the database. The inference monitor can prioritize the base stations according to the amount of interference symbols and their signal strengths. Then the monitor gives a base station interference severity list. The technician can execute interference analysis tool that can point out the interfering CPEs individually. Furthermore, the interference analysis tool has very good visualization function which is presented in detail in the next slide.

The outputs of interference monitoring and analyzer are the valuable input for frequency optimization. For instance, based on the amount of symbols, signal strength and location of the individual CPEs, technicians can select the best optimization method to reduce interference, i.e. tilt antennas, changing the direction of the antenna or correct the frequency etc. 


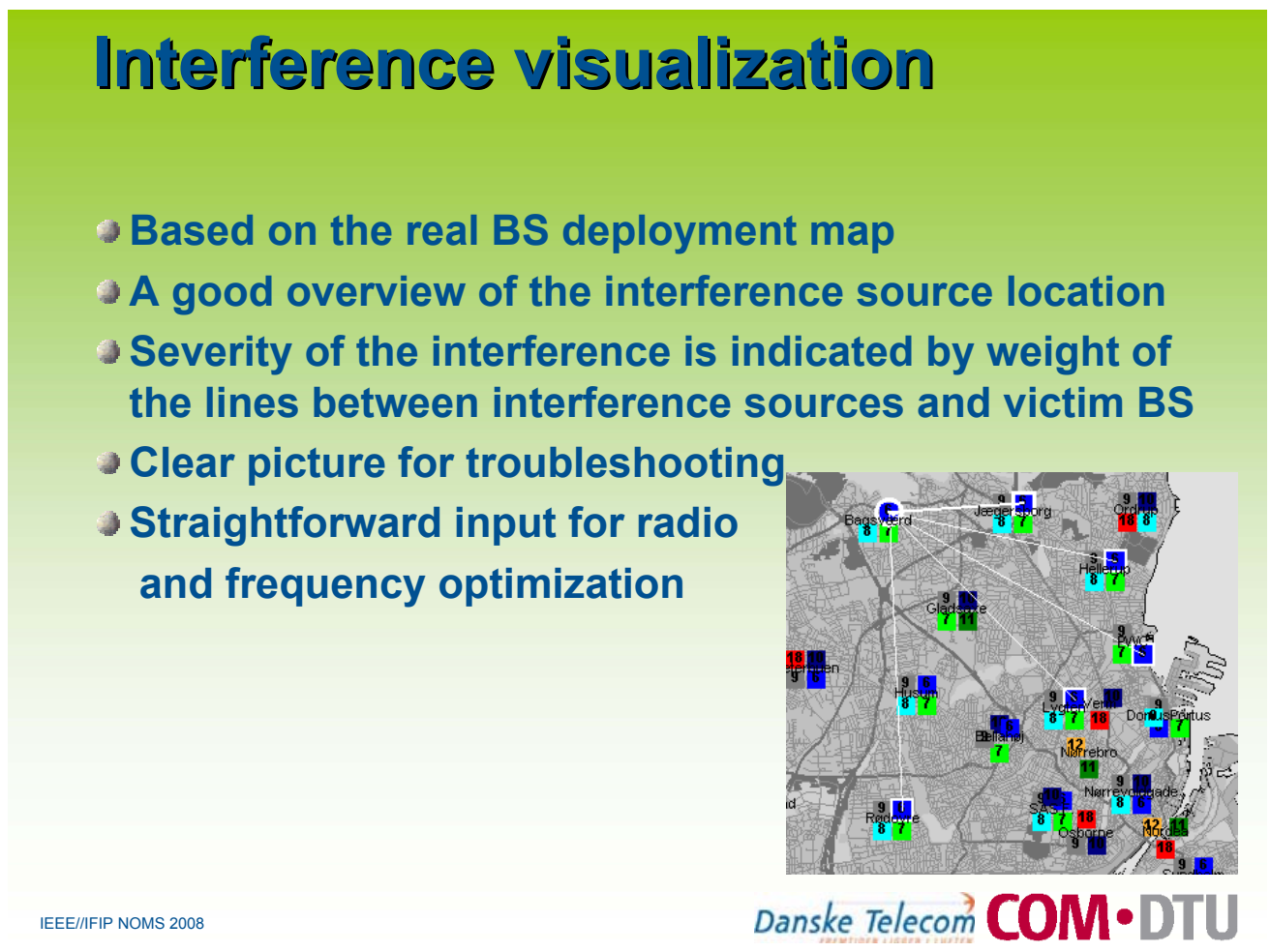

The uplink interference visualization is shown on the map which is marked with all the base station location and the corresponding frequency. For each victim base station, it can provide a good overview of the interfering source CPE location. The severity of the interference is differentiated by the weight of the lines between the interference source and victim BS. The interference visualization gives a clear picture for troubleshooting and a straightforward input for radio frequency optimization. For instance, when a base station has received high interference, it is easy to find out who brings about the interference. Then according to the map, we can analyze the reason of the interference whether it is because of too short frequency reuse distance, because of special landscape, or because of base station antenna has improper tilt, etc. 


\section{Report Generation \& Alarm}

management

- Report Generation

$>$ Predefined automatically generated report: daily, weekly, monthly

$>$ On-demand report

\section{- Alarm management}

> Performance alarm for the various exceptional network performance, e.g.

- Packet loss rate is higher threshold

- Error symbol rate is higher than threshold

- ...

- Alarms for the operation of the Monitoring tool, e.g. collector is down

- Inform the administrator by email alert

- Error report is stored as log file

Our system has two different ways to generate performance reports. One is predefined periodical report which is programmed into the tool and the report generator can automatically send daily, weekly and monthly performance reports. The other is the report generated based on demands. This type of report is triggered by technical support through the user interface of the tool, which can show the the selected performance with arbitrary duration.

Alarm management is another important function module. All the alarms are sent by email to the technicians. The alarm can be categorized into two classes: performance alarm and operation alarm.

The performance alarm alerts the technicians to the various exceptional network performance by comparing to the predefined performance thresholds. For instance, the packet loss rate, the error symbol rate are higher than their thresholds.

The operation alarm is for the operation of the monitoring tools. Because the monitoring tool is still under development and test, some exceptions might happen to the software. There is a logging service which keeps an eye on the program and logs the error or exception in the program. It will inform the administrator by email in case of program exception, for instance, the data collector is down. It also equips the developer with detailed context for application failures, which can be used to debug the program later. Furthermore, to some extent operation alarm also relates to the network operation. For instance, in the case of base station is down and the data collector cannot get performance data from the network periodically, it also gives the alarm. 


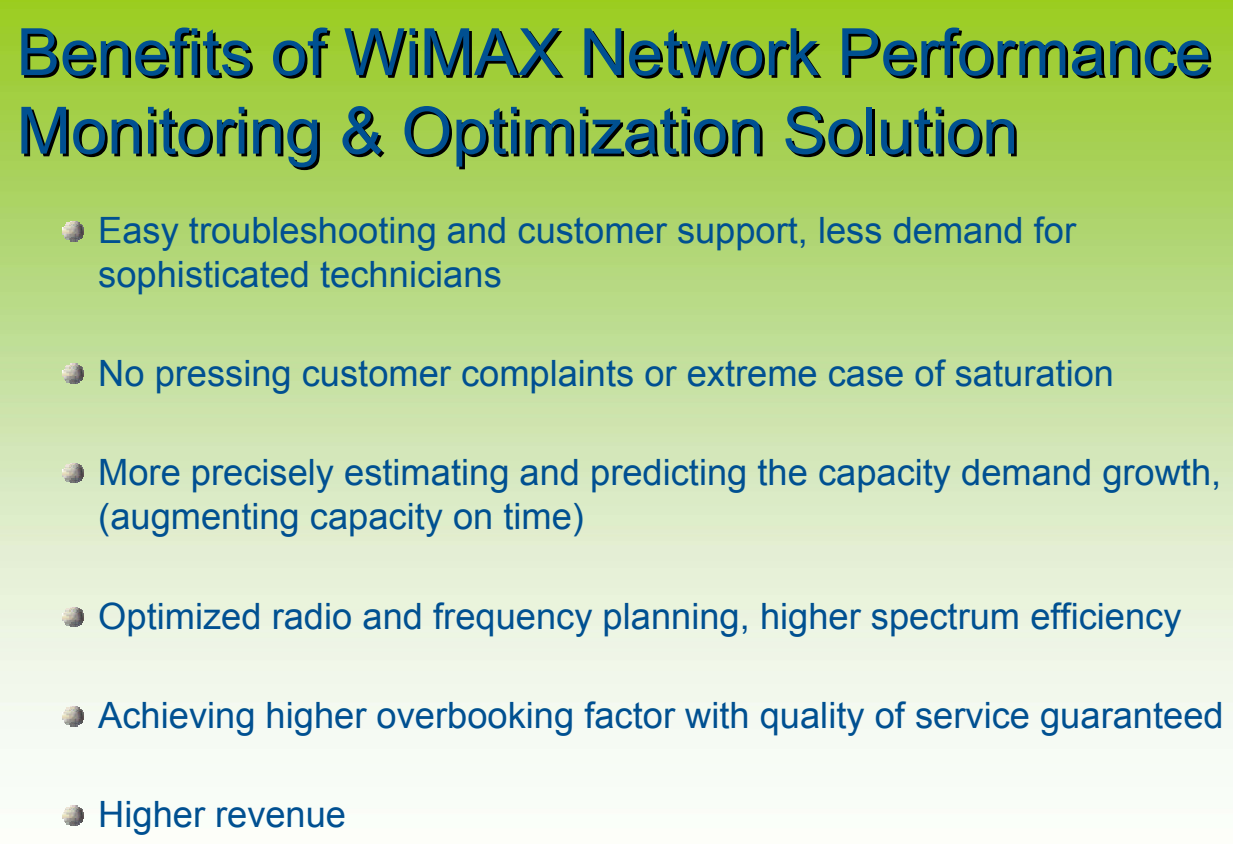

Optimized radio and frequency planning, higher spectrum efficiency

- Achieving higher overbooking factor with quality of service guaranteed

- Higher revenue

IEEE/IIFIP NOMS 2008

\section{Danske Telecom COM・DTU}

The main benefits of the integrated WiMAX network performance monitoring and optimization solution are list as following. It makes troubleshooting and customer support much easier, therefore there is less demand for sophisticated technicians. For example, when customer support technician receive customer complaint calls, they can immediately check the CPE traffic usage, CPE radio signal quality and CPE roaming graph during specific time duration. The analysis results can give customer support technician a very straight-forward picture of the CPE's statuses. For instance, radio signal quality sudden deterioration is often because that user moves the CPE to a bad location at the house. With the assistant of dynamic QoS control tool, there are no pressing customer complaints or extreme case of saturation. It also greatly saves man hours. Furthermore, the results from saturation monitoring, DQC and CPE mobility tracking tools are valuable to precisely estimate and predict the capacity demand growth. A case in point, when saturation occurs in the BS, it might be because of abusive users or that the network capacity is not enough. So if the gain from DQC reaction becomes smaller, it indicates that it is the time to increase the network capacity. With radio signal quality tool and interference tool, it can optimize the radio and frequency planning using the right optimization method. Thus the higher spectrum efficiency is achieved in our network. The whole solution makes it possible to achieve high overbooking factor meanwhile guarantees QoS. From business perspective, the integrated performance monitoring and optimization system reduces the CAPEX and OPEX, which brings higher revenue for the wireless broadband operator. 